\title{
Interaction of half-quantized vortices in two-component Bose-Einstein condensates
}

\author{
Minoru Eto ${ }^{1}$, Kenichi Kasamatsu ${ }^{2}$, Muneto $\mathrm{Nitta}^{3}$, Hiromitsu Takeuchi ${ }^{4}$, and Makoto Tsubota ${ }^{4}$ \\ ${ }^{1}$ Theoretical Physics Laboratory, RIKEN, Saitama 351-0198, Japan \\ ${ }^{2}$ Department of Physics, Kinki University, Higashi-Osaka, 577-8502, Japan \\ ${ }^{3}$ Department of Physics, and Research and Education Center for Natural Sciences, \\ Keio University, Hiyoshi 4-1-1, Yokohama, Kanagawa 223-8521, Japan \\ ${ }^{4}$ Department of Physics, Osaka City University, Sumiyoshi-Ku, Osaka 558-8585, Japan
}

(Dated: October 16, 2018)

\begin{abstract}
We study the asymptotic interaction between two half-quantized vortices in two-component BoseEinstein condensates. When two vortices in different components are placed at distance $2 R$, the leading order of the force between them is found to be $(\log R / \xi-1 / 2) / R^{3}$, in contrast to $1 / R$ between vortices placed in the same component. We derive it analytically using the Abrikosov ansatz and the profile functions of the vortices, confirmed numerically with the Gross-Pitaevskii model. We also find that the short-range cutoff of the inter-vortex potential linearly depends on the healing length.

PACS numbers: 03.75.Lm, 03.75.Mn, 11.25.Uv, 67.85.Fg
\end{abstract}

\section{INTRODUCTION}

Multicomponent condensations appear in many systems in condensed matter physics and QCD, from multi-component or spinor Bose-Einstein condensates (BECs), superfluid ${ }^{3} \mathrm{He}$, multi-gap superconductors to chiral phase transition or color superconductors in QCD at high temperature and/or high density. Especially, multicomponent and spinor BECs admit a rich variety of topological excitations: Domain walls [1], Abelian [2] and non-Abelian [3] vortices, monopoles [4], 2D Skyrmions [5], 3D Skyrmions [6, 7], vortons [8], knots [9], and Dbrane solitons [10]. See [2, 11, 12] as a review. Among these topological excitations, quantized vortices in multicomponent BECs are the most important subject, because they are closely related to the problems not only in other condensed matter systems such as superconductors, superfluids, magnetism, and liquid crystal, but also in electro-weak theory [13], QCD and grand unified theories in high energy physics, neutron stars and cosmic strings in cosmology [14, 15].

Interactions between quantized vortices are important information to determine the equilibrium configuration and dynamics of many vortices. It is known that, in a single-component BEC, the asymptotic interaction energy per unit length of two parallel vortex lines separated by a distance $R$ is proportional to $\log (L / R)$, where $L$ is the size of the system [16]. Thus, the inter-vortex force has $1 / R$ dependence. Vortices in a BEC resemble with global vortices in relativistic field theories [17]-[20]. A relation between them was studied in [20] where it was suggested that spinning global vortices on a Lorentz violating background behave as superfluid vortices. Global vortices are regarded as global cosmic strings or axion strings in cosmology and the inter-vortex force between two global vortices was shown to be $1 / R[18$ ], coinciding with the one in vortices in a scalar BEC, scalar superfluid, and the XY model. Global vortices also appear in $\mathrm{QCD}$; in chiral phase transition of QCD at high temper- ature or high density 21, 22] or color superconductor of extremely high density QCD [23, 24]. Inter-vortex force at large distance $R$ was derived analytically at the leading order as $1 / R$ for color superconductor [24], and $\cos \alpha / R$ with a relative orientation $\alpha$ of two vortices in the internal space for chiral phase transition [22]; see [25] as a review.

However, the analytic formula of the vortex-vortex interactions in multicomponent BECs are still missing. Two-component BECs are the simplest example of the multicomponent condensates and have also attracted much interest to study the novel phenomena not found in a single component BEC. Recent experiments provide a good ground of study on the vortex-vortex interaction in two-component BECs by tuning s-wave scattering length via a Feshbach resonance [26-28]. The minimally quantized vortex in two-component BECs has the winding number one half of a singly-quantized vortex in scalar BECs, and thus is often called a half-quantized vortex. Its mass circulation is fractionally quantized when mass densities of two condensates are different. Such a quantized vortex in two-component BECs has a composite structure, where a vortex core in one component is filled by the density of the other component. This vortex structure was created experimentally through coherent interconversion between two components 29]. Interactions between the vortices in the different components are nontrivial because the two components interact only through the density, so that the vortex winding around one component does not directly experience the circulation of the other vortex winding around the other component. This fact results in an indirect interaction, where the filling component of each vortex core is affected by the circulation created by the vortex in the same component, dragging the vortex in which it is filled. Although the interactive dynamics of two vortices in two-component BECs was studied numerically by Öhberg and Santos [30], the analytical form of the interaction force was not discussed.

In this paper, we consider the asymptotic interaction 
between two vortices in two-component BECs. We consider the vortex-vortex interaction for two cases (i) two vortices are placed in the different components and (ii) those in the same component. For the case (i), the leading order of the inter-vortex force between them at distance $2 R$ is found to be $(\log R / \xi-1 / 2) / R^{3}$ with the short-range cutoff $\xi$, in contrast to the one $1 / R$ for the case (ii) and vortices in a single-component BEC. We derive it analytically using the Abrikosov ansatz and the asymptotic profile functions of the vortices. We then confirm it numerically. We also find that the short-range cutoff $\xi$ of the inter-vortex potential linearly depends on the healing length.

This paper is organized as follows. Sec. II is devoted for deriving the analytic form of the asymptotic intervortex force. In Sec. III we confirm the analytic results obtained in Sec. II by numerical calculations of the GrossPitaevskii equation. Summary and discussions are in Sec. IV. In Appendix, we describe some details of the calculation of integrals in Sec. II C.

\section{STATIC INTER-VORTEX FORCES}

\section{A. The model}

We start with an energy functional for two-component BEC system

$$
\begin{aligned}
& E\left(\Psi_{1}, \Psi_{2}\right)=K\left(\Psi_{1}, \Psi_{2}\right)+V\left(\Psi_{1}, \Psi_{2}\right), \\
& K=\int d^{3} x \sum_{i=1,2}\left(-\frac{\hbar^{2}}{2 m_{i}} \Psi_{i}^{*} \nabla^{2} \Psi_{i}\right) \\
& V=\int d^{3} x\left[\sum_{i=1,2} \frac{g_{i}}{2}\left|\Psi_{i}\right|^{4}+g_{12}\left|\Psi_{1}\right|^{2}\left|\Psi_{2}\right|^{2}\right]
\end{aligned}
$$

where $\Psi_{i}$ is a condensate wave function of the $i$-th component $(i=1,2)$ with mass $m_{i}$. The coupling constants $g_{1}, g_{2}$ and $g_{12}$ stand for the atom-atom interactions; the $\Psi_{1}$ and $\Psi_{2}$ components repel or attract for $g_{12}>0$ or $g_{12}<0$, respectively. The coupled Gross-Pitaevskii (GP) equations are obtained by the variational principle $i \hbar \partial_{t} \Psi_{i}=\delta E / \delta \Psi_{i}^{*}$ as

$$
\begin{aligned}
& i \hbar \partial_{t} \Psi_{1}=\left(-\frac{\hbar^{2} \nabla^{2}}{2 m_{1}}+g_{1}\left|\Psi_{1}\right|^{2}+g_{12}\left|\Psi_{2}\right|^{2}\right) \Psi_{1} \\
& i \hbar \partial_{t} \Psi_{2}=\left(-\frac{\hbar^{2} \nabla^{2}}{2 m_{2}}+g_{2}\left|\Psi_{2}\right|^{2}+g_{12}\left|\Psi_{1}\right|^{2}\right) \Psi_{2}
\end{aligned}
$$

The stationary coupled GP equation is given by considering a time dependence $\Psi_{i}(\mathbf{x}, t)=e^{-i \mu_{i} t / \hbar} \Psi_{i}(\mathbf{x})$ with the chemical potential $\mu_{i}$

$$
\begin{aligned}
& \left(-\frac{\hbar^{2} \nabla^{2}}{2 m_{1}}-\mu_{1}+g_{1}\left|\Psi_{1}(\mathbf{x})\right|^{2}+g_{12}\left|\Psi_{2}(\mathbf{x})\right|^{2}\right) \Psi_{1}(\mathbf{x})=0 \\
& \left(-\frac{\hbar^{2} \nabla^{2}}{2 m_{2}}-\mu_{2}+g_{2}\left|\Psi_{2}(\mathbf{x})\right|^{2}+g_{12}\left|\Psi_{1}(\mathbf{x})\right|^{2}\right) \Psi_{2}(\mathbf{x})=0 .
\end{aligned}
$$

The potential energy $V$ with the quadratic terms $-\mu_{1}\left|\Psi_{1}\right|^{2}-\mu_{2}\left|\Psi_{2}\right|^{2}$ induced by the chemical potential is a quadratic function of $X \equiv\left|\Psi_{1}\right|^{2} \geq 0$ and $Y \equiv\left|\Psi_{2}\right|^{2} \geq 0$,

$$
V(X, Y)=\frac{g_{1}}{2} X^{2}+\frac{g_{2}}{2} Y^{2}+g_{12} X Y-\mu_{1} X-\mu_{2} Y .
$$

Let $g_{1}, g_{2}$ be positive, then the potential $V$ has a minimum when

$$
\begin{array}{r}
\Delta \equiv V_{X X} V_{Y Y}-V_{X Y}^{2}=g_{1} g_{2}-g_{12}^{2}>0 \\
\mu_{1} g_{2}-\mu_{2} g_{12} \geq 0, \quad \mu_{2} g_{1}-\mu_{1} g_{12} \geq 0
\end{array}
$$

The amplitudes of the ground state are then given by

$$
\begin{aligned}
& \left|\Psi_{1}\right|=\sqrt{\frac{\mu_{1} g_{2}-\mu_{2} g_{12}}{g_{1} g_{2}-g_{12}^{2}}} \equiv v_{1}, \\
& \left|\Psi_{2}\right|=\sqrt{\frac{\mu_{2} g_{1}-\mu_{1} g_{12}}{g_{1} g_{2}-g_{12}^{2}}} \equiv v_{2} .
\end{aligned}
$$

In the following, we consider the situation in which the above inequalities are satisfied. Since there are two condensates, two $U(1)$ symmetries are spontaneously broken. Accordingly the order parameter space is

$$
T^{2} \simeq U(1)_{1} \times U(1)_{2} \simeq \frac{U(1)_{\mathrm{mass}} \times U(1)_{\mathrm{spin}}}{\mathbb{Z}_{2}} .
$$

Here each $U(1)_{i}(i=1,2)$ corresponds to the phase rotation of $\Psi_{1}$ or $\Psi_{2}$, while $U(1)_{\text {mass }}$ and $U(1)_{\text {spin }}$ correspond to the overall and relative phase rotations, defined by

$$
\begin{array}{rll}
U(1)_{\text {mass }}: & \Psi_{1} \rightarrow \Psi_{1} e^{i \alpha}, & \Psi_{2} \rightarrow \Psi_{2} e^{i \alpha}, \\
U(1)_{\text {spin }}: & \Psi_{1} \rightarrow \Psi_{1} e^{i \beta}, & \Psi_{2} \rightarrow \Psi_{2} e^{-i \beta},
\end{array}
$$

whose currents are mass and pseudo-spin currents, respectively. Both the condensates $\Psi_{1}, \Psi_{2}$ are unchanged under the $\mathbb{Z}_{2}$ action $(\alpha=\beta=\pi)$ inside $U(1)_{\text {mass }} \times$ $U(1)_{\text {spin }}$ in Eq. (12), and therefore this $\mathbb{Z}_{2}$ has to be removed, as the denominator of Eq. (11).

In what follows, we call the phase cycles for $\Psi_{1}$ and $\Psi_{2}$ the $(1,0)$ - and $(0,1)$-cycles, respectively.

\section{B. Vortex configuration}

Since the first homotopy group of order parameter space is

$$
\pi_{1}\left(T^{2}\right)=\mathbb{Z} \oplus \mathbb{Z}
$$


it allows two kinds of winding numbers. We refer a vortex winding around $(1,0)[(0,1)]$-cycle once as a $(1,0)$ vortex $[(0,1)$-vortex $]$, which is the most fundamental vortex. When one travels around a $(1,0)[(0,1)]$-vortex, the phase of $\Psi_{1}\left(\Psi_{2}\right)$ rotates by $2 \pi$ with the phase of the other component kept constant. On the other hand, in terms of $U(1)_{\text {mass }}$ and $U(1)_{\text {spin }}$ in Eq. (12), $U(1)_{\text {mass }}$ is rotated by $\pi$ and $U(1)_{\text {spin }}$ is rotated by $+\pi(-\pi)$ with circling around a $(1,0)[(0,1)]$-vortex. Since they have a half winding of $U(1)_{\text {mass }}$, they are often called half-quantized vortices.

Vortices winding around both components by $2 \pi$ are denoted by $(1,1)$ and have unit winding in $U(1)_{\text {mass }}$.
They are called integer vortices, if the core is not separated into $(1,0)$ and $(0,1)$ vortices. More generally we refer a configuration which winds $(1,0)$-cycle $m$ times and $(0,1)$-cycle $n$ times as an $(m, n)$-vortex, whose wave function is denoted as $\Psi_{i}^{(m, n)}$ for $i$-th component.

The vortex configuration can be obtained by solving Eqs. (6) and (77). Let us make an ansatz for an axially symmetric $(1,0)$-vortex configuration

$$
\Psi_{1}^{(1,0)}=v_{1} e^{i \theta} f_{(1,0)}(r), \quad \Psi_{2}^{(1,0)}=v_{2} h_{(1,0)}(r),
$$

where $r$ and $\theta$ are the polar coordinates.

The profile functions $f_{(1,0)}$ and $h_{(1,0)}$ are determined by substituting (14) into (6) and (7), as

$$
\begin{gathered}
-\frac{\hbar^{2}}{2 m_{1}}\left(f_{(1,0)}^{\prime \prime}+\frac{f_{(1,0)}^{\prime}}{r}-\frac{f_{(1,0)}}{r^{2}}\right)+\frac{\mu_{1} g_{1} g_{2}\left(f_{(1,0)}^{2}-1\right)-\mu_{1} g_{12}^{2}\left(h_{(1,0)}^{2}-1\right)-\mu_{2} g_{1} g_{12}\left(f_{(1,0)}^{2}-h_{(1,0)}^{2}\right)}{g_{1} g_{2}-g_{12}^{2}} f_{(1,0)}=0, \\
-\frac{\hbar^{2}}{2 m_{2}}\left(h_{(1,0)}^{\prime \prime}+\frac{h_{(1,0)}^{\prime}}{r}\right)+\frac{\mu_{2} g_{1} g_{2}\left(h_{(1,0)}^{2}-1\right)-\mu_{2} g_{12}^{2}\left(f_{(1,0)}^{2}-1\right)-\mu_{1} g_{2} g_{12}\left(h_{(1,0)}^{2}-f_{(1,0)}^{2}\right)}{g_{1} g_{2}-g_{12}^{2}} h_{(1,0)}=0,
\end{gathered}
$$

with the prime denoting a differentiation with respect to $r$. We solve these equations with the boundary conditions

$$
\begin{aligned}
& \left(f_{(1,0)}, h_{(1,0)}\right) \rightarrow(1,1) \quad \text { as } \quad r \rightarrow \infty, \\
& \left(f_{(1,0)}, h_{(1,0)}^{\prime}\right) \rightarrow(0,0) \quad \text { as } r \rightarrow 0 .
\end{aligned}
$$

From these equations, asymptotic behaviors of the profile functions $f_{(1,0)}$ and $h_{(1,0)}$ at large distance can be obtained as

$$
\begin{aligned}
& f_{(1,0)}(r)=1-\frac{1}{m_{1} \eta_{1}^{+} r^{2}}+\mathcal{O}\left(r^{-4}\right), \\
& h_{(1,0)}(r)=1+\frac{1}{m_{1} \eta_{1}^{-} r^{2}}+\mathcal{O}\left(r^{-4}\right),
\end{aligned}
$$

where we have introduced the effective mass parameters

$$
\eta_{1}^{+} \equiv \frac{4\left(\mu_{1} g_{2}-\mu_{2} g_{12}\right)}{g_{2} \hbar^{2}}, \quad \eta_{1}^{-} \equiv \frac{4\left(\mu_{2} g_{1}-\mu_{1} g_{12}\right)}{g_{12} \hbar^{2}} .
$$

The stability condition Eq. (9) of the ground state ensures that $\eta_{1}^{+}>0$, while $\eta_{1}^{-}$changes its sign with $g_{12}$.

Similarly, we make an ansatz for the $(0,1)$-vortex

$$
\Psi_{1}^{(0,1)}=v_{1} h_{(0,1)}(r), \quad \Psi_{2}^{(0,1)}=v_{2} e^{i \theta} f_{(0,1)}(r) .
$$

The equations for $f_{(0,1)}, h_{(0,1)}$ can be obtained by just replacing the indices as $1 \leftrightarrow 2$ and $(1,0) \leftrightarrow(0,1)$ in Eqs. (15) and (16). Then the asymptotic behaviors are

$$
\begin{aligned}
& f_{(0,1)}(r)=1-\frac{1}{m_{2} \eta_{2}^{+} r^{2}}+\mathcal{O}\left(r^{-4}\right), \\
& h_{(0,1)}(r)=1+\frac{1}{m_{2} \eta_{2}^{-} r^{2}}+\mathcal{O}\left(r^{-4}\right),
\end{aligned}
$$

with

$$
\eta_{2}^{+} \equiv \frac{4\left(\mu_{2} g_{1}-\mu_{1} g_{12}\right)}{g_{1} \hbar^{2}}, \quad \eta_{2}^{-} \equiv \frac{4\left(\mu_{1} g_{2}-\mu_{2} g_{12}\right)}{g_{12} \hbar^{2}} .
$$

Again, $\eta_{2}^{+}$is always positive while sign of $\eta_{2}^{-}$depends on $g_{12}$.

As vortices in a scalar BEC, the tension (energy per unit length) of $(1,0)$ and $(0,1)$ vortices logarithmically diverges as

$$
T_{(1,0)} \simeq \frac{\pi \hbar^{2} v_{1}^{2}}{m_{1}} \log \frac{L}{\xi}, \quad T_{(0,1)} \simeq \frac{\pi \hbar^{2} v_{2}^{2}}{m_{2}} \log \frac{L}{\xi},
$$

respectively, with $L$ and $\xi$ being a long and short distance cutoff, respectively. This divergent behavior comes from the kinetic term in the GP energy functional Eq. (3).

Some numerical solutions of the single vortex configurations are shown in Fig. 1. A universal feature of configuration is that $h_{(1,0)}$ (the profile function of unwinding field) at the vortex center is concave for $g_{12}<0$ and convex for the $g_{12}>0$ [31]. This can be understood from the atom-atom interaction $g_{12}$; in the presence of the vortex profile for $\Psi_{1}$ as a background, $\Psi_{2}$ feels the potential $g_{12}\left|\Psi_{1}\right|^{2}$ and it tends to be trapped in the vortex center for the repulsive interaction $g_{12}>0$ and to be exclusive from the vortex center for the attractive interaction $g_{12}<0$.

\section{Inter-vortex forces}

It is expected that the interactions between $(1,0)$ - and $(0,1)$-vortices are determined by the coupling $g_{12}$-term . 

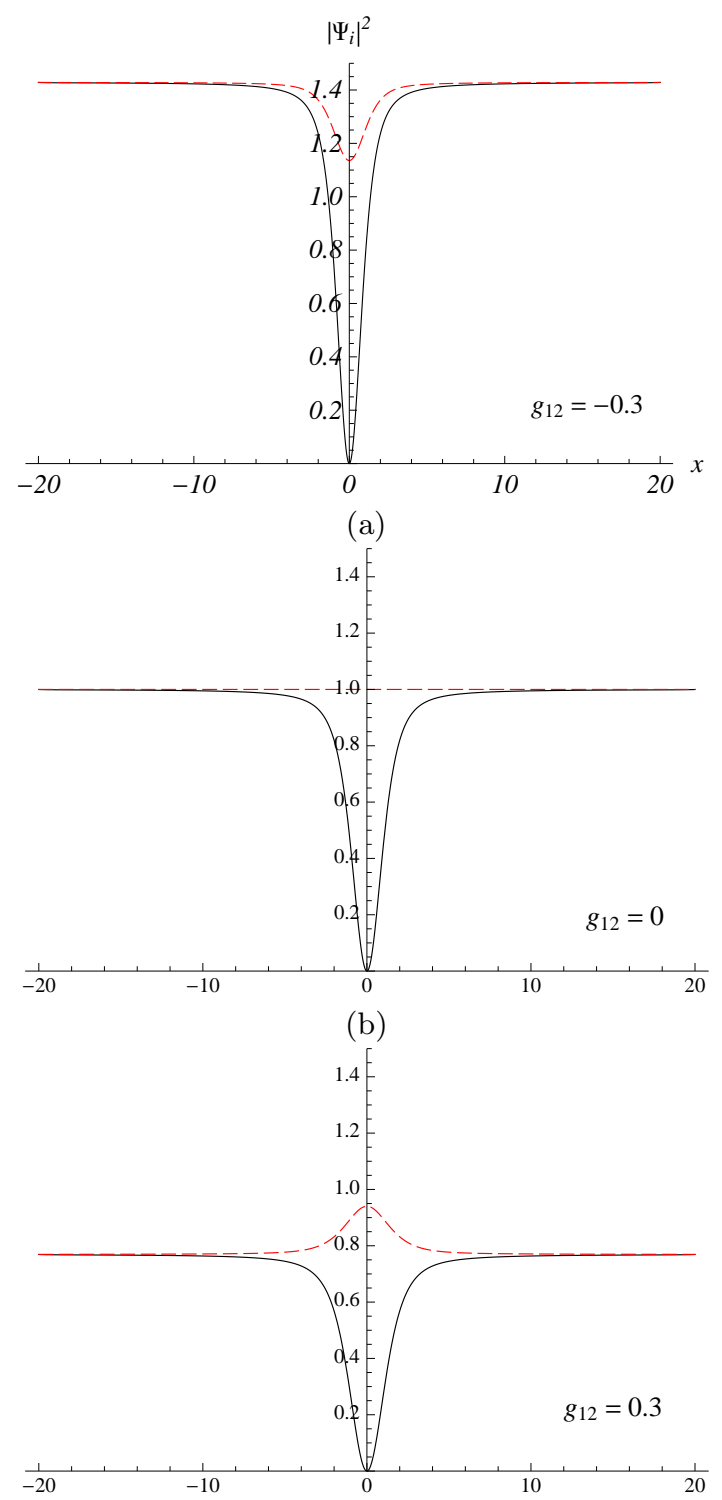

(c)
When $g_{12}$ is zero, they are decoupled in Eqs. (4) and (5), so $(1,0)$ and $(0,1)$ vortices do not interact. Here, we calculate the asymptotic interactions between well separated $(1,0)$ - and $(0,1)$-vortices using their asymptotic profile functions obtained in the last subsection. Let us place the $(1,0)$ - and $(0,1)$-vortices at $(x, y)=(R, 0)$ and $(x, y)=(-R, 0)$, respectively as in Fig. 2, We use the polar coordinates $(r, \theta)$ with the origin $(x, y)=(0,0)$. We further express $\left(r_{(1,0)}, \theta_{(1,0)}\right)$ and $\left(r_{(0,1)}, \theta_{(0,1)}\right)$ as the

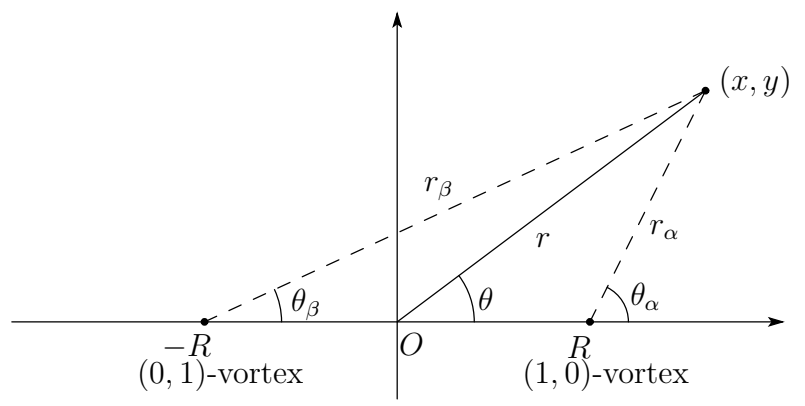

FIG. 2: Configuration of $(1,0)$-vortex and $(0,1)$-vortex.

polar coordinates with the origins at the $(1,0)$ and $(0,1)$ vortex centers $(R, 0)$ and $(-R, 0)$, respectively. Then the following relations hold among three polar coordinates

$$
\begin{aligned}
& r_{i}^{2}=(r \cos \theta \mp R)^{2}+r^{2} \sin ^{2} \theta, \\
& \tan \theta_{i}=\frac{r \sin \theta}{r \cos \theta \mp R}
\end{aligned}
$$

with $i=(1,0),(0,1)$, the minus sign for $i=(1,0)$ and the plus sign for $i=(0,1)$. With these coordinates, the $(1,0)$ - and $(0,1)$-vortex configurations $\left(\Psi_{1}^{(1,0)}, \Psi_{2}^{(1,0)}\right)$ and $\left(\Psi_{1}^{(0,1)}, \Psi_{2}^{(0,1)}\right)$ can be expressed as

$$
\begin{aligned}
& \Psi_{1}^{(1,0)}=v_{1} e^{i \theta_{(1,0)}} f_{(1,0)}\left(r_{(1,0)}\right), \\
& \Psi_{2}^{(1,0)}=v_{2} h_{(1,0)}\left(r_{(1,0)}\right), \\
& \Psi_{1}^{(0,1)}=v_{1} h_{(0,1)}\left(r_{(0,1)}\right), \\
& \Psi_{2}^{(0,1)}=v_{2} e^{i \theta_{(0,1)}} f_{(0,1)}\left(r_{(0,1)}\right) .
\end{aligned}
$$
-0.3 , (b) $g_{12}=0$ and (c) $g_{12}=0.3$.

Let us now calculate the interaction between $(1,0)$-vortex and $(0,1)$-vortex. We first make the standard Abrikosov ansatz

$$
\begin{aligned}
& \Psi_{1}^{(1,1)}(r, \theta)=v_{1}^{-1} \Psi_{1}^{(1,0)} \Psi_{1}^{(0,1)} \simeq v_{1}\left(1-\frac{1}{m_{1} \eta_{1}^{+} r_{(1,0)}^{2}}+\frac{1}{m_{2} \eta_{2}^{-} r_{(0,1)}^{2}}\right) e^{i \theta_{(1,0)}}+\mathcal{O}\left(r^{-4}\right), \\
& \Psi_{2}^{(1,1)}(r, \theta)=v_{2}^{-1} \Psi_{2}^{(1,0)} \Psi_{2}^{(0,1)} \simeq v_{2}\left(1-\frac{1}{m_{2} \eta_{2}^{+} r_{(0,1)}^{2}}+\frac{1}{m_{1} \eta_{1}^{-} r_{(1,0)}^{2}}\right) e^{i \theta_{(0,1)}}+\mathcal{O}\left(r^{-4}\right)
\end{aligned}
$$

for the total configuration. 
Then the interaction potential is obtained by subtracting two individual vortex energies from the total energy as

$$
U_{(1,1)}=\int d^{2} x(\delta K+\delta V)
$$

where it has two contributions: one from the kinetic energy $\delta K=K\left(\Psi_{1}^{(1,1)}, \Psi_{2}^{(1,1)}\right)-K\left(\Psi_{1}^{(1,0)}, \Psi_{2}^{(1,0)}\right)-$ $K\left(\Psi_{1}^{(0,1)}, \Psi_{2}^{(0,1)}\right)$ and the other from the potential energy $\delta V=V\left(\Psi_{1}^{(1,1)}, \Psi_{2}^{(1,1)}\right)-V\left(\Psi_{1}^{(1,0)}, \Psi_{2}^{(1,0)}\right)-$ $V\left(\Psi_{1}^{(0,1)}, \Psi_{2}^{(0,1)}\right)+V\left(v_{1}, v_{2}\right)$.

By using the asymptotic properties given in Eqs. (19), (20), (23), (24), (30) and (31), we find

$$
\begin{aligned}
\delta K= & \frac{v_{1}^{2} \hbar^{2}}{m_{1} m_{2} \eta_{2}^{-} r_{(0,1)}^{2}}\left(\nabla \theta_{(1,0)}\right)^{2} \\
& +\frac{v_{2}^{2} \hbar^{2}}{m_{1} m_{2} \eta_{1}^{-} r_{(1,0)}^{2}}\left(\nabla \theta_{(0,1)}\right)^{2}+\mathcal{O}\left(r^{-6}\right) \\
= & \frac{g_{12} \hbar^{4}}{2 m_{1} m_{2}\left(g_{1} g_{2}-g_{12}^{2}\right) r_{(1,0)}^{2} r_{(0,1)}^{2}}+\mathcal{O}\left(r^{-6}\right)
\end{aligned}
$$

where we have used $\left(\nabla \theta_{(1,0)}\right)^{2}=r_{(1,0)}^{-2},\left(\nabla \theta_{(0,1)}\right)^{2}=$ $r_{(0,1)}^{-2}$ and have taken terms up to $\mathcal{O}\left(r^{-4}\right)$. It is important to see that the leading terms of the order $\mathcal{O}\left(r^{-2}\right)$ have been canceled out in the subtraction. Therefore, the dominant contribution to the interaction potential is of the order $\mathcal{O}\left(r^{-4}\right)$. Similarly, we find the terms of the order $\mathcal{O}\left(r^{-4}\right)$ in the potential energy

$$
\delta V=-\frac{g_{12} \hbar^{4}}{4 m_{1} m_{2}\left(g_{1} g_{2}-g_{12}^{2}\right) r_{(1,0)}^{2} r_{(0,1)}^{2}}+\mathcal{O}\left(r^{-6}\right)
$$

Plugging these into Eq. (32), we get

$$
\begin{aligned}
U_{(1,1)}(R) & =\frac{g_{12} \hbar^{4}}{4 m_{1} m_{2}\left(g_{1} g_{2}-g_{12}^{2}\right)} \int d^{2} x \frac{1}{r_{(1,0)}^{2} r_{(0,1)}^{2}} \\
& \simeq \frac{g_{12} \hbar^{4} \pi}{4 m_{1} m_{2}\left(g_{1} g_{2}-g_{12}^{2}\right)} \frac{\log \frac{R}{\xi}}{R^{2}}
\end{aligned}
$$

where $\xi$ stands for a short distance cut-off and we have used $R \gg \xi$. The detailed calculation of Eq. (35) is described in Appendix. Here, the terms independent of $R$ have been ignored. The factor $1 / R^{2}$ is a striking feature which is absent in the scalar BEC or scalar superfluids. Note that the chemical potentials $\mu_{1}$ and $\mu_{2}$ do not appear in the final result (35). The asymptotic force between the two vortices is obtained by differentiating the potential by their distance $2 R$, as $F_{(1,1)}(R)=-\frac{d U_{(1,1)}}{2 d R}$,

$$
F_{(1,1)}(R)=\frac{\pi \hbar^{4} g_{12}}{4 m_{1} m_{2}\left(g_{1} g_{2}-g_{12}^{2}\right)} \frac{1}{R^{3}}\left(\log \frac{R}{\xi}-\frac{1}{2}\right) .
$$

We have found that the interaction is attractive for $g_{12}<$ 0 , repulsive for $g_{12}>0$ and vanishes for $g_{12}=0$.

Note that the asymptotic interaction is independent of the sign of the vortex winding number $e^{ \pm i \theta}$, because the interaction between the two condensates is mediated only through their amplitudes as $g_{12}\left|\Psi_{1}\right|^{2}\left|\Psi_{2}\right|^{2}$. In fact, the interaction potential $U_{(1,-1)}$ between $(1,0)$ and $(0,-1)$ vortices are exactly the same as $U_{(1,1)}$. It is easy to verify that the following relation holds

$$
U_{(1,1)}=U_{(1,-1)}=U_{(-1,1)}=U_{(-1,-1)} .
$$

This is because $\theta_{(1,0)}$ and $\theta_{(0,1)}$ are decoupled in the Abrikosov ansatz in Eqs. (30) and (31).

The potential (35) should be compared with the potential $U_{(1 \pm 1,0)}$ between $(1,0)$ and $( \pm 1,0)$ vortices. To see it, we make the ordinary Abrikosov ansatz

$$
\Psi_{1}^{(1 \pm 1,0)} \simeq v_{1} e^{i\left(\theta_{(1,0)} \pm \theta_{(0,1)}\right)}, \quad \Psi_{2}^{(1 \pm 1,0)} \simeq v_{2} .
$$

Note here that we have taken terms of the order unity. A leading order contribution to the interaction comes from the kinetic term of $\Psi_{1}$ which is of order $\mathcal{O}\left(r^{-2}\right)$. On the other hand, the kinetic energy of $\Psi_{2}$ and the potential energy contributions start from the order $\mathcal{O}\left(r^{-4}\right)$, so we omit them. The interaction potential is then given by

$$
\begin{aligned}
U_{(1 \pm 1,0)} & = \pm \frac{v_{1}^{2} \hbar^{2}}{m_{1}} \int d^{2} x \vec{\nabla} \theta_{(1,0)} \cdot \vec{\nabla} \theta_{(0,1)} \\
& = \pm \frac{\left(\mu_{1} g_{2}-\mu_{2} g_{12}\right) \hbar^{2} \pi}{\left(g_{1} g_{2}-g_{12}^{2}\right) m_{1}} \log \frac{R^{2}+L^{2}}{4 R^{2}}
\end{aligned}
$$

where $L$ is an infrared cut-off parameter; see Appendix for the details. Unlike the case of the leading term in the potential (35) between $(1,0)$ and $(0,1)$ vortices, the potential $U_{(1 \pm 1,0)}$ depends on the chemical potential. We also note that it depends on the infrared cutoff $L$ but not on the ultraviolet cutoff $\xi$.

The inter-vortex force $F_{(1 \pm 1,0)}=-\frac{d U_{(1 \pm 1,0)}}{2 d R}$ is then

$$
\begin{aligned}
F_{(1 \pm 1,0)} & = \pm \frac{\left(\mu_{1} g_{2}-\mu_{2} g_{12}\right) \hbar^{2} \pi}{\left(g_{1} g_{2}-g_{12}^{2}\right) m_{1}}\left(\frac{1}{R}-\frac{R}{R^{2}+L^{2}}\right) \\
& \rightarrow \pm \frac{\left(\mu_{1} g_{2}-\mu_{2} g_{12}\right) \hbar^{2} \pi}{\left(g_{1} g_{2}-g_{12}^{2}\right) m_{1}} \frac{1}{R}
\end{aligned}
$$

where $\rightarrow$ denotes the large volume limit $L \rightarrow \infty$. This $1 / R$ force is well known for vortices in the scalar BEC, scalar superfluids and the XY model, and global vortices in relativistic field theories [16, 18]. The correction term for finite volume $L$ can be found in the second term in the brace in the first line. This term might not be so familiar but has been obtained previously in [22] for global nonAbelian vortices in QCD.

In the same way, the interaction potential between $(0,1)$ - and $(0, \pm 1)$-vortices are given by

$$
U_{(0,1 \pm 1)}(R)= \pm \frac{\left(\mu_{2} g_{1}-\mu_{1} g_{12}\right) \hbar^{2} \pi}{\left(g_{1} g_{2}-g_{12}^{2}\right) m_{2}} \log \frac{R^{2}+L^{2}}{4 R^{2}}
$$

\section{NUMERICAL ANALYSIS}

Let us numerically verify the interaction potential analytically obtained in Eqs. (35). For simplicity, we consider a special case $m_{1}=m_{2}=m, g_{1}=g_{2}=g$ and 


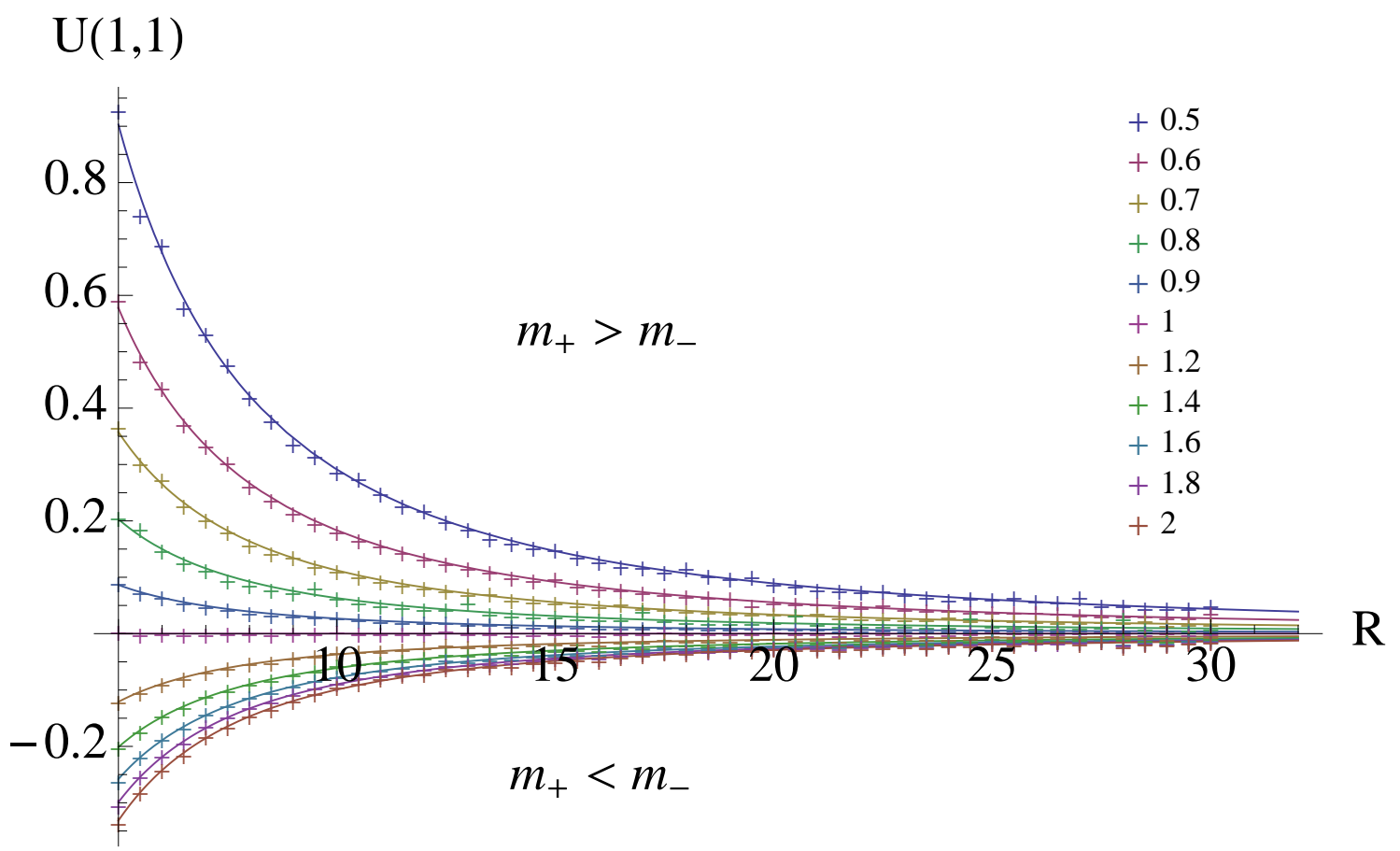

FIG. 3: The inter-vortex potential $U_{(1,1)}(R)$ for $m_{-}=(0.5,0.6,0.7,0.8,0.9,1,1.2,1.4,1.6,1.8,2)$ with $m_{+}=1$. Solid lines are asymptotic inter-vortex forces (Abrikosov ansatz) which are analytically obtained in Eq. (35).

$\mu_{1}=\mu_{2}=\mu$. Then the asymptotic behaviors of the profile functions in Eqs. (19) and (20) are rewritten as follows,

$$
\begin{aligned}
f_{i}+h_{i} & =2-\frac{1}{m_{+}^{2} r^{2}}+\mathcal{O}\left(r^{-4}\right), \\
f_{i}-h_{i} & =-\frac{1}{m_{-}^{2} r^{2}}+\mathcal{O}\left(r^{-4}\right)
\end{aligned}
$$

for $i=(1,0)$ and $(0,1)$, where the mass parameters $m_{+}$ and $m_{-}$are defined by

$$
m_{+}^{2} \equiv \frac{4 m \mu}{\hbar^{2}}, \quad m_{-}^{2} \equiv \frac{4 m \mu}{\hbar^{2}} \frac{g-g_{12}}{g+g_{12}}
$$

The inverse numbers of $m_{+}$and $m_{-}$give the healing lengths associated with the mass component $f_{i}+h_{i}$ and the spin component $f_{i}-h_{i}$, respectively. The inter-vortex potential Eq. (35) is then expressed as

$$
U_{(1,1)}(R)=\frac{\pi}{2} v^{2} \frac{m_{+}^{2}-m_{-}^{2}}{m_{+}^{2} m_{-}^{2}} \frac{1}{R^{2}} \log \frac{R}{\xi}
$$

where $v^{2}=\hbar^{2} \mu / m\left(g+g_{12}\right)$ is defined in Eq. (10).

To obtain the inter-vortex potential numerically, we use a sort of the imaginary time propagation of the GP equation as

$$
\begin{array}{r}
\left(-\frac{\hbar^{2}}{2 m} \nabla^{2}-\mu+g\left|\Psi_{1}(\mathbf{x})\right|^{2}+g_{12}\left|\Psi_{2}(\mathbf{x})\right|^{2}\right) \Psi_{1}(\mathbf{x}, \tau) \\
=-D_{1}(\mathbf{x}) \partial_{\tau} \Psi_{1}(\mathbf{x}, \tau), \\
\left(-\frac{\hbar^{2}}{2 m} \nabla^{2}-\mu+g\left|\Psi_{2}(\mathbf{x})\right|^{2}+g_{12}\left|\Psi_{1}(\mathbf{x})\right|^{2}\right) \Psi_{2}(\mathbf{x}, \tau) \\
=-D_{2}(\mathbf{x}) \partial_{\tau} \Psi_{2}(\mathbf{x}, \tau),
\end{array}
$$

where $\tau$ is the imaginary time and $D_{1}$ and $D_{2}$ are positive coefficients. While $D_{i}$ is set to be a constant in the usual imaginary time propagation, we consider the coefficient $D_{i}=D_{i}(\mathbf{x})$ with space-coordinate dependence. An advantage of using $D_{i}(\mathbf{x})$ is that one can effectively fix the position of vortex (the zeros of $\Psi_{i}$ ) during the numerical calculation, if one choose $D_{i}(\mathbf{x})$ appropriately. In order to attain this, we choose a function $D_{i}(\mathbf{x})=A \nabla^{2} \log \left(\left|\mathbf{x}-\mathbf{a}_{i}\right|^{2}+\epsilon^{2}\right)+c$ where $\mathbf{a}_{i}$ stands for the $i$-th vortex position and $A$ and $c$ are positive constants. The value of $A$ is taken as an extremely large value to fix the profile of the wave function only near the vortex cores. Also, $\epsilon$ should be sufficiently small. We chose $A=80000, \epsilon=0.01$ and $c=0.1$ in our numerical computation. We take the Abrikosov ansatz given in Eqs. (30) and (31) as the initial condition at $\tau=0$ and minimize the energy under the imaginary time evolution. After the solutions converge sufficiently, we calculate the interaction energy Eq. (32).

Throughout our numerical computation below, we will set $m / \hbar^{2}=1$ and $v^{2}=1$. Then we regard $m_{+}$and $m_{-}$as 
independent parameters of the GP equations and perform the numerical calculation by varying them. Remember that $m_{+}<m_{-}$corresponds to $g_{12}<0$ (attractive force) whereas $m_{+}>m_{-}$corresponds to $g_{12}>0$ (repulsive force). No net interaction exists accidentally when $m_{+}=$ $m_{-}$.

The result is shown in Fig. 3. We compare the intervortex potential obtained numerically and the one obtained analytically. As can be seen, the analytic results reproduce the numerical results quite well. We have only one fitting parameter $2 \xi$ which is the the short-range cutoff. The values $2 \xi$ for various choice of $m_{-}$for fixed $m_{+}=1$ are shown in Fig. 4. We find the linear dependence of the short-range cut-off $\xi$ on the healing length $1 / m_{-}$.

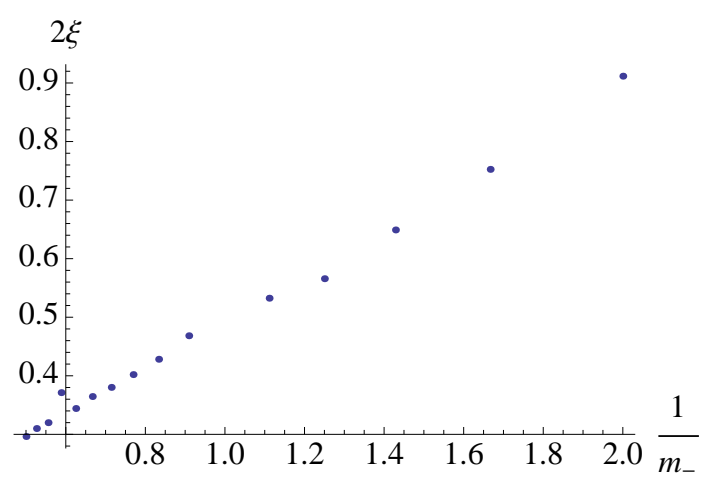

FIG. 4: The dependence of the healing length $2 \xi$ on $1 / m_{-}$ with fixed $m_{+}=1$.

\section{SUMMARY AND DISCUSSION}

We have studied the asymptotic interaction between half-quantized vortices, i.e., $(1,0)$ and $(0, \pm 1)$ vortices winding around $\Psi_{1}$ and $\Psi_{2}$, respectively in the twocomponent BEC. Since the two components interact only through the density, the $(1,0)$-vortex does not directly experience the circulation of the $(0, \pm 1)$-vortex so that the result does not depend on the signature of the winding number. The leading order of the force between them is found to be $\sim[\log (R / \xi)-1 / 2] / R^{3}$ in contrast to the one between the same kind of vortices $\sim 1 / R$, which is also well known as the force between vortices in scalar BEC, scalar superfluid and the XY model, and global vortices in relativistic field theories. We have first derived it analytically using the Abrikosov ansatz and the asymptotic profile functions of $(1,0)$ - and $(0,1)$-vortices. We have then confirmed it numerically with using the extended imaginary time method for the GP equations. We have found that the short-range cut-off parameter $\xi$ of the vortex interaction linearly depends on the healing length $1 / m_{-}$.

Our results suggest a bound state of $(1,0)$ and $(0, \pm 1)$ vortices for $g_{12}<0$. While a set of $(1,0)$ and $(0,1)$ is expected to form a stable integer (mass) vortex $(1,1)$, it is a nontrivial question if $(1,0)$ and $(0,-1)$ vortices form a bound state, which should be called a (pseudo-) spin vortex. Also, one expects no stable bound states for $g_{12}>0$. Although there should be instabilities for large separation at least, it does not exclude a possibility of a metastable bound state at short distance. To address these questions, we need to know a short range interaction or stability analysis of the bound states, which remains as a future problem.

Multiple vortices will constitute a vortex lattice in experiments of multicomponent BECs under the rotation 32]. Ample phase diagram of the vortex lattices was predicted in [33] and was numerically obtained in [2, 34]. Since we have obtained the analytic expression of the inter-vortex forces, we expect to explain the vortex phase diagram analytically. Especially, we expect that the difference of the force $[\log (R / \xi)-1 / 2] / R^{3}$ between $(1,0)$ and $(0,1)$ vortices and the one $1 / R$ between the same kind of vortices will determine it.

Our method should be extended to spinor BECs, which remains as an interesting future problem. On the other hand multicomponent systems in relativistic field theories are common in QCD such as the linear sigma model for the chiral phase transition and the Landau-Ginzburg model for color superconductors at high baryon density 23]. In these models, order parameters are matrices as in superfluid ${ }^{3} \mathrm{He}$ rather than vectors, and consequently there exist non-Abelian vortices [35]: non-Abelian global vortices in the chiral phase transition [21] and nonAbelian semi-superfluid vortices in color superconductors at high baryon density [23]. Inter-vortex forces have been calculated at leading order for non-Abelian global vortices 22] and non-Abelian semi-superfluid vortices [24], see [25] for a review. Calculation in the present paper will give the next leading order $[\log (R / \xi)-1 / 2] / R^{3}$ to them. Especially the force $\cos \alpha / R$ between non-Abelian global vortices at the leading order vanishes for a particular choice $(\alpha= \pm \pi)$ of internal orientations of vortices [22], and therefore the next leading order term proportional to $[\log (R / \xi)-1 / 2] / R^{3}$ becomes a dominant contribution. An extension of our results to these cases should be important to consider a possibility of vortex lattice phases in heavy-ion collisions or in a neutron star core, as in two-component BEC [2, 33, 34].

\section{Acknowledgments}

The work of M.E. is supported by Special Postdoctoral Researchers Program at RIKEN. The work of K.K. and M.N. is supported in part by Grant-in-Aid for Scientific Research (Grant No. 21740267 (K.K.) and No. 20740141(M.N.)) from MEXT, Japan. 


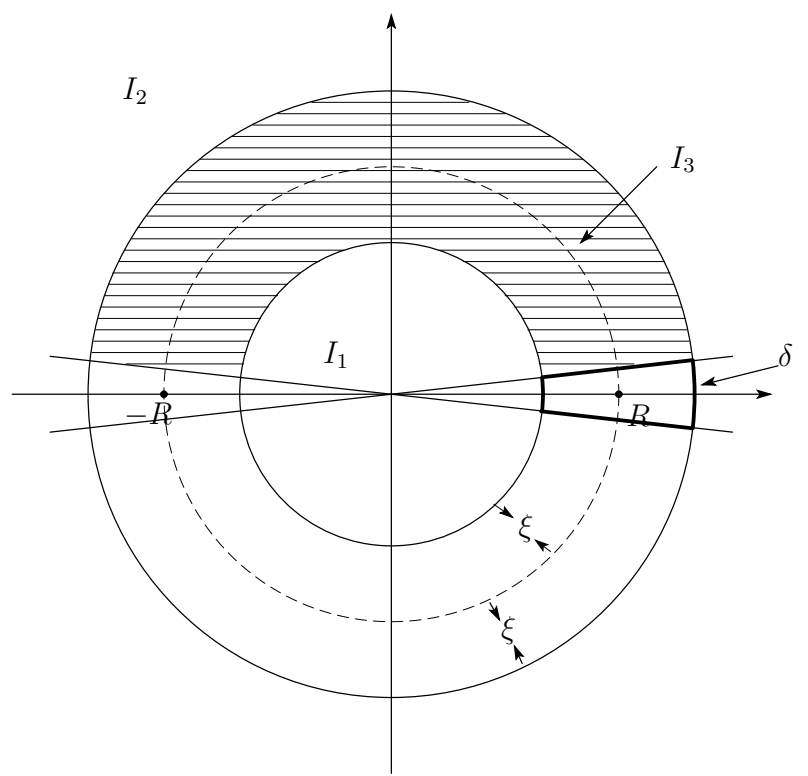

FIG. 5: The integral region to calculate Eq. A2

\section{Appendix A: Derivation of Eqs. (35) and (39)}

In Eq. (35), we have to evaluate the integral

$$
\begin{aligned}
I & =\int d^{2} x \frac{1}{r_{(1,0)}^{2} r_{(0,1)}^{2}}=\int d^{2} x \frac{1}{A_{0}}, \\
A_{0} & \equiv r^{4}+R^{4}-2 r^{2} R^{2} \cos 2 \theta .
\end{aligned}
$$

To this end we will use a formula

$$
\int_{0}^{2 \pi} d \theta \frac{1}{A+B \cos 2 \theta}=\frac{2 \pi}{\sqrt{A^{2}-B^{2}}}
$$

for $A>|B|$. To evaluate Eq. (A2), we divide the integral region as shown in Fig. 5. In addition to $I_{1}$ and $I_{2}$, we take into account the contributions $I_{3}$ from the strip of width $2 \xi$. Since the integrand diverges at $(x, y)=$ $( \pm R, 0)$, we introduce an ultraviolet cut-off $\xi$. Then, we will remove the small regions that includes the points of vortex positions. Hence, the total integral is written as

$$
I_{\text {cut off }}=I_{1}+I_{2}+2 I_{3} \text {. }
$$

For $R \gg \xi$ the integral $I_{1}$ and $I_{2}$ is calculated as

$$
\begin{aligned}
& I_{1}=\int_{0}^{R-\xi} \frac{2 \pi r d r}{R^{4}-r^{4}} \simeq \frac{\pi}{2 R^{2}}\left(\log \frac{R}{\xi}+\mathcal{O}\left(\frac{\xi}{R}\right)\right) \\
& I_{2}=\int_{R+\xi}^{\infty} \frac{2 \pi r d r}{R^{4}-r^{4}} \simeq \frac{\pi}{2 R^{2}}\left(\log \frac{R}{\xi}+\mathcal{O}\left(\frac{\xi}{R}\right)\right),
\end{aligned}
$$

where we have used Eq. (A3). The remaining integral

$$
I_{3}=\int_{R-\xi}^{R+\xi} d r \int_{\delta}^{\pi-\delta} d \theta \frac{r}{A_{0}}
$$

with $\xi / R \ll 1$ and $\delta \ll 1$ is evaluated as follows. Note that $\cos 2 \theta \leq \cos 2 \delta=1-2 \delta^{2}+\cdots<1-\delta^{2}$ and $A_{0}>$ $\left(r^{2}-R^{2}\right)^{2}+2 r^{2} R^{2} \delta^{2} \geq 2 r^{2} R^{2} \delta^{2}$. Thus, we have the following inequality

$$
0 \leq I_{3} \leq \frac{\pi-2 \delta}{2 R^{2} \delta^{2}} \log \frac{R+\xi}{R-\xi} \simeq \frac{\pi}{R^{2} \delta^{2}} \frac{\xi}{R}
$$

Thus, for any $\delta$, one can choose sufficiently small $\xi$, so that $I_{3}$ becomes negligibly small.

In summary, we get

$$
I_{\text {cut }- \text { off }}=\frac{\pi}{R^{2}}\left(\log \frac{R}{\xi}+\mathcal{O}\left(\frac{\xi}{R}\right)\right) .
$$

Next, we calculate the integration in Eq. (39),

$$
\begin{aligned}
J & =\int d^{2} x \vec{\nabla} \theta_{(1,0)} \cdot \vec{\nabla} \theta_{(0,1)}=\int d^{2} x \frac{r^{2}-R^{2}}{r_{(1,0)}^{2} r_{(0,1)}^{2}} \\
& =\int d r d \theta \frac{r\left(r^{2}-R^{2}\right)}{r^{4}+R^{4}-2 r^{2} R^{2} \cos 2 \theta}
\end{aligned}
$$

By using Eq. (A3), one can first perform the integration in $\theta$ and then integrate with respect $r$ as

$$
\begin{aligned}
J & =\int_{0}^{\infty} d r \frac{2 \pi r\left(r^{2}-R^{2}\right)}{\sqrt{\left(r^{4}-R^{4}\right)^{2}}} \\
& =\lim _{L \rightarrow \infty}\left[-\int_{0}^{R} \frac{2 \pi r d r}{r^{2}+R^{2}}+\int_{R}^{L} \frac{2 \pi r d r}{r^{2}+R^{2}}\right] \\
& =\lim _{L \rightarrow \infty} \pi \log \frac{L^{2}+R^{2}}{4 R^{2}} .
\end{aligned}
$$

[1] T. L. Ho and V. B. Shenoy, Phys. Rev. Lett. 77, 5276 (1996); E. Timmermans, Phys. Rev. Lett. 81, 5718 (1998); B. V. Schaeybroeck, Phys. Rev. A 78, 023624 (2008).

[2] K. Kasamatsu, M. Tsubota and M. Ueda, Int. J. Mod. Phys. B 19, 1835 (2005).

[3] G. W. Semenoff and F. Zhou, Phys. Rev. Lett. 98, 100401 (2007); M. Kobayashi, Y. Kawaguchi, M. Nitta and M. Ueda, Phys. Rev. Lett. 103, 115301 (2009).
[4] H. T. C. Stoof, E. Vliegen and U. Al Khawaja, Phys. Rev. Lett. 87, 120407 (2001); J. -P. Martikainen, A. Collin, and K.-A. Suominen, Phys. Rev. Lett. 88, 090404 (2002); C. M. Savage and J. Ruostekoski, Phys. Rev. A 68, 043604 (2003).

[5] T. L. Ho, Phys. Rev. Lett. 81, 742 (1998); U. Leonhardt, G. E. Volovik, JETP Lett. 72, 46 (2000). A. E. Leanhardt, Y. Shin, D. Kielpinski, D. E. Pritchard, and W. Ketterle, Phys. Rev. Lett. 90, 140403 (2003). 
[6] J. Ruostekoski, and J. R. Anglin, Phys. Rev. Lett. 86, 3934 (2001); R. A. Battye, N. R. Cooper, and P. M. Sutcliffe, Phys. Rev. Lett. 88, 080401 (2002); C. M. Savage and J. Ruostekoski, Phys. Rev. Lett. 91, 010403 (2003).

[7] U. A. Khawaja and H. T. C. Stoof, Nature (London) 411, 918 (2001), Phys. Rev. A 64, 043612 (2001).

[8] M. A. Metlitski and A. R. Zhitnitsky, JHEP 0406, 017 (2004); J. Ruostekoski, Phys. Rev. A 70, 041601(R) (2004);

[9] Y. Kawaguchi, M. Nitta, and M. Ueda, Phys. Rev. Lett. 100, 180403 (2008).

[10] K. Kasamatsu, H. Takeuchi, M. Nitta and M. Tsubota, JHEP 11, 068 (2010).

[11] M. Ueda and Y. Kawaguchi, arXiv:1001.2072 (2010).

[12] Y. Kawaguchi, M. Kobayashi, M. Nitta and M. Ueda, Prog. Theor. Phys. Suppl. 186, 455 (2010).

[13] A. Achucarro and T. Vachaspati, Phys. Rept. 327, 347 (2000)

[14] M. B. Hindmarsh and T. W. B. Kibble, Rept. Prog. Phys. 58, 477 (1995)

[15] A. Vilenkin and E. P. S. Shellard, Cosmic Strings and Other Topological Defects, (Cambridge Univ. Press,1994).

[16] C. J. Pethick and H. Smith, Bose-Einstein Condensation in Dilute Gases, 2nd ed. (Cambridge University Press, New York, 2008).

[17] A. Vilenkin and A. E. Everett, Phys. Rev. Lett. 48, 1867 (1982).

[18] E. P. S. Shellard, Nucl. Phys. B 283, 624 (1987).

[19] L. Perivolaropoulos, Nucl. Phys. B 375, 665 (1992).

[20] R. L. Davis and E. P. S. Shellard, Phys. Rev. Lett. 63, 2021 (1989); R. L. Davis, Phys. Rev. D 40, 4033 (1989); B. A. Gradwohl, G. Kaelbermann, T. Piran and E. Bertschinger, Nucl. Phys. B 338, 371 (1990).

[21] A. P. Balachandran and S. Digal, Phys. Rev. D 66, 034018 (2002); M. Nitta and N. Shiiki, Phys. Lett. B 658, 143 (2008); M. Eto, E. Nakano and M. Nitta, Nucl. Phys. B 821, 129 (2009).

[22] E. Nakano, M. Nitta and T. Matsuura, Phys. Lett. B 672, 61 (2009).

[23] A. P. Balachandran, S. Digal and T. Matsuura, Phys.
Rev. D 73, 074009 (2006); M. Eto and M. Nitta, Phys. Rev. D 80, 125007 (2009); M. Eto, E. Nakano and M. Nitta, Phys. Rev. D 80, 125011 (2009); M. Eto, M. Nitta and N. Yamamoto, Phys. Rev. Lett. 104, 161601 (2010); Phys. Rev. D (in press) arXiv:1101.2574 [hep-ph]]; Y. Hirono, T. Kanazawa and M. Nitta, Phys. Rev. D (in press) arXiv:1012.6042 [hep-ph]]; S. Yasui, K. Itakura and M. Nitta, Phys. Rev. D 81, 105003 (2010). Phys. Rev. B (in press) arXiv:1010.3331 [cond-mat.meshall]].

[24] E. Nakano, M. Nitta and T. Matsuura, Phys. Rev. D 78, 045002 (2008).

[25] E. Nakano, M. Nitta and T. Matsuura, Prog. Theor. Phys. Suppl. 174, 254 (2008).

[26] G. Thalhammer, G. Barontini, L. De Sarlo, J. Catani, F. Minardi, and M. Inguscio, Phys. Rev. Lett. 100, 210402 (2008).

[27] S. B. Papp, J. M. Pino, and C. E. Wieman, Phys. Rev. Lett. 101, 040402 (2008).

[28] S. Tojo, Y. Taguchi, Y. Masuyama, T. Hayashi, H. Saito, and T. Hirano, Phys. Rev. A 82, 033609 (2010).

[29] M. R. Matthews, B. P. Anderson, P. C. Haljan, D. S. Hall, C. E. Wieman, and E. A. Cornell, Phys. Rev. Lett., 83, 2498 (1999).

[30] P. Öhberg and L. Santons, Phys. Rev. A 66, 013616 (2002).

[31] This behaviour is the same for global non-Abelian vortices appearing in the chiral phase transition of QCD, see the second reference in [21]. Similar holds for semisuperfluid non-Abelian vortices in color superconductors of QCD at high density, see the second reference in 23].

[32] V. Schweikhard, I. Coddington, P. Engels, S. Tung, and E. A. Cornell, Phys. Rev. Lett. 93, 210403 (2004).

[33] E. J. Mueller and T. L. Ho Phys. Rev. Lett. 88, 180403 (2002).

[34] K. Kasamatsu, M. Tsubota and M. Ueda, Phys. Rev. Lett. 91, 150406 (2003).

[35] The terminology "non-Abelian vortices" here denotes vortices in symmetry breaking of $G$ to $H$ with a nonAbelian group $H$ and $\pi_{1}(G / H) \neq 0$. 